\title{
Evaluación neuropsicológica en niños con epilepsia: atención y funciones ejecutivas en epilepsia del lóbulo temporal
}

\author{
Ana F.D. Lopes, Mário M.R. Simões, Conceiçao N. Robalo, Isabel Fineza, Olavo B. Gonçalves
}

Introducción. La atención y las funciones ejecutivas se corresponden con áreas de funciones cognitivas asociadas al lóbulo frontal. El estudio de la atención y las funciones ejecutivas en niños con epilepsia se ha enfocado en caracterizar pacientes afectos de epilepsia del lóbulo frontal, aunque recientemente también se han identificado déficit en esas áreas en epilepsia del lóbulo temporal.

Objetivo. Investigar la atención y las funciones ejecutivas en niños con epilepsia del lóbulo temporal, considerando también la influencia de variables clínicas (edad de comienzo, evolución de la epilepsia).

Sujetos y métodos. Se estudió la atención y las funciones ejecutivas en 24 niños afectos de epilepsia del lóbulo temporal, de entre 7 y 15 años, y se compararon con 24 niños controles. A todos ellos se les pasaron las siguientes pruebas: test de cancelación, Trail Making Test, torre de Londres y fluencia verbal fonémica.

Resultados. El grupo con epilepsia del lóbulo temporal rendía sustancialmente por debajo tanto en atención sostenida y dividida como en fluencia verbal fonémica. En los marcadores que evaluaban omisiones y errores, no había diferencias entre los diferentes grupos en ninguno de los test aplicados. Se comprobó también un enlentecimiento de la capacidad de procesamiento. Además, los pacientes con mayor precocidad de inicio de la epilepsia presentaban mayores dificultades en el mantenimiento de la atención y en las capacidades de planificación.

Conclusiones. Estos resultados refuerzan la necesidad de evaluar y monitorizar el área de la atención, de las funciones ejecutivas y de la velocidad de procesamiento en niños con epilepsia del lóbulo temporal, sobre todo en los que tienen un comienzo precoz de la epilepsia.

Palabras clave. Atención. Epilepsia del lóbulo temporal. Evaluación neuropsicológica. Fluencia verbal fonémica. Funciones ejecutivas. Niños. Test de cancelación. Torre de Londres. Trail Making Test.

\section{Introducción}

Una de las funciones que pueden alterarse en las personas con epilepsia es la atención [1-4], independientemente del nivel de las funciones intelectuales [5]. También son frecuentes las quejas por falta de atención por parte de padres y profesores de los pacientes con epilepsia [6]. Se calcula que la tercera parte de los niños epilépticos sufre problemas de atención [7]. Probablemente, entre los factores que más contribuyen a desarrollar problemas de atención se encuentren la disfunción bioeléctrica, los tratamientos farmacológicos y la disfunción cerebral subyacente [8-10].

La atención es uno de los procesos básicos del funcionamiento cognitivo, y es el que asegura la conexión entre la percepción y el procesamiento de la información [11]. Dentro de la palabra 'atención' se incluyen varios apartados: el estado de vigilancia -forma básica de la atención (el nivel de alerta o de vigilancia) que expresa la disponibilidad fisiológica para procesar la información y su respuesta correspondiente-, la atención selectiva (la capacidad de enfoque en un estímulo determinado), la atención dividida (la capacidad de prestar atención simultáneamente a más de un estímulo) y la atención sostenida (la capacidad para mantener la atención durante un período prolongado). La atención, por su parte, también influencia y modula la realización de otras funciones cognitivas, cooperando con algunas de ellas, generalmente con la percepción y con las funciones ejecutivas, con las que a menudo es erróneamente confundida.

Se denominan funciones ejecutivas una serie de funciones heterogéneas que abarcan diversos aspectos del proceso cognitivo, como planificación, organización, autorregulación, inhibición y flexibilidad [12-15], y su función principal es la de guiar la
Servicio de Evaluación Psicológica; Facultad de Psicología y Ciencias de la Educación; Centro de Psicopedagogía; Universidad de Coimbra (A.F.D. Lopes, M.M.R. Simões). Centro de Desarrollo Infantil Luís Borges; Hospital Pediátrico de Coimbra (C.N. Robalo, I. Fineza, O.B. Gonçalves). Coimbra, Portugal.

Correspondencia: Dra. Ana Filipa Lopes. Servicio de Evaluación Psicológica. Facultad de Psicología y Ciencias de la Educación. Universidad de Coimbra. Rua do Colégio Novo. Apdo. 6153. 3001-802 Coimbra, Portugal.

E-mail:

anafilipalopes@fpce.uc.pt

Aceptado tras revisión externa: 26.10.09.

Cómo citar este artículo: Lopes AFD, Simões MMR, Robalo $\mathrm{CN}$, Fineza I, Gonçalves OB. Evaluación neuropsicológica en niños con epilepsia:atención y funciones ejecutivas en epilepsia del lóbulo temporal. Rev Neurol 2010; 50: 265-72.

(C) 2010 Revista de Neurología 
adaptación individual a nuevas situaciones. Baron [16] define las funciones ejecutivas como aptitudes metacognitivas que permiten al sujeto percibir los estímulos, responder de forma adaptada, cambiar direcciones con flexibilidad, anticipar objetivos futuros, considerar consecuencias y responder de forma conjunta para usar todas estas capacidades para conseguir un objetivo común.

Los déficit de atención y de funcionamiento ejecutivo son valiosos predictores del rendimiento escolar. Un estudio de Williams et al [17] ha encontrado más conexiones significativas entre atención y fracaso escolar que con otras variables (memoria y factores socioeconómicos). No obstante, en algunos niños pueden considerarse las dificultades de atención como la primera advertencia de que existe un déficit cognitivo causado por las convulsiones [18].

Se considera la atención y las funciones ejecutivas como unas de las funciones principales asumidas por la corteza frontal [19]. No obstante, el estudio de la atención y, sobre todo, de las funciones ejecutivas en niños y adolescentes epilépticos se ha enfocado preferentemente en caracterizar a los pacientes con epilepsia del lóbulo frontal. Sin embargo, recientes estudios han registrado déficit de estas áreas en niños afectados de epilepsia del lóbulo temporal [4,20-26]. Estas alteraciones pueden explicarse de dos maneras: las funciones ejecutivas tienen una disfunción subyacente de las conexiones frontotemporales [24] y el hipocampo puede intervenir en algunas áreas de las funciones ejecutivas [27].

En los niños con epilepsia del lóbulo temporal, la gravedad de la disfunción de la atención y de las funciones ejecutivas parece estar relacionada con algunas variables propias de la epilepsia, como una edad precoz de comienzo, una mayor duración de la epilepsia y el uso de politerapia [23,28].

La finalidad de este trabajo es la investigación de la función neuropsicológica, especialmente de la atención y de las funciones ejecutivas, en un grupo de niños y adolescentes con epilepsia del lóbulo temporal, considerando también la influencia de otras variables clínicas (edad de comienzo de la epilepsia y evolución de las crisis).

\section{Sujetos y métodos}

El presente estudio se realizó en el Centro de Desarrollo Infantil Luís Borges, en el Hospital Pediátrico de Coimbra. Se pidió a todos los padres completar un formulario de consentimiento informado para que los niños tomaran parte en el estudio.

El protocolo de evaluación incluía:
- Entrevista con los padres del niño o del adolescente para saber su nivel de rendimiento escolar y la marcha de su desarrollo, así como información respecto al curso de la epilepsia (las historias clínicas de cada uno de ellos fueron también consultadas y se contactó con el neuropediatra a su cargo siempre que se consideró necesario).

- Test de inteligencia para niños de Wechsler, tercera edición (WISC-III) [29].

- Pruebas de atención y de funciones ejecutivas de la batería de evaluación neuropsicológica de Coimbra [30].

Los instrumentos para atención y funciones ejecutivas fueron los siguientes:

- Test de cancelación. Valora la atención sostenida. Consiste en dos folios A3 con 1.600 cuadrados (40 líneas, cada una con 40 cuadrados). De ellos, sólo 10 (test de cancelación con dos signos) o 15 (test de cancelación con tres signos) corresponden a uno de los cuadrados modelo. La base de la prueba es trazar una línea sobre los cuadrados objetivo durante 10 minutos. Hay dos formas del test: una para niños entre 6 y 9 años (dos signos) y otra para adolescentes de 10 a 15 años (tres signos).

- Trail Making Test. Valora la atención selectiva (parte A), la atención dividida (parte B) y la velocidad de procesamiento motor. El test consta de dos partes diferentes, A y B. En la parte A, el sujeto debe usar un lápiz para dibujar una línea entre 25 círculos numerados (distribuidos aleatoriamente en una hoja de papel), siguiendo el orden correcto entre 1 y 25 . En la parte B, el sujeto debe dibujar una línea entre 25 círculos que contienen números o letras, también distribuidos aleatoriamente en orden alterno entre los números 1 y 13, alternando con letras (conectando 1 con A, A con 2, 2 con B, B con 3, etc.).

- Torre de Londres. Valora las funciones ejecutivas, como planificación, control, autorregulación y capacidades para resolver problemas. El niño tiene que replicar la torre en de 12 a 14 modelos diferentes, moviendo tres esferas de colores, siguiendo tres reglas: primera regla, cada eje de torre sólo contiene un número determinado de esferas; segunda regla, sólo puede moverse una esfera cada vez; y tercera regla, cada problema permite un número de movimientos limitado para realizar el modelo presentado en la tarjeta correspondiente. Cuando se falta a alguna de estas reglas, se considera que el sujeto ha cometido errores de tipo 1 , de tipo 2 y de tipo 3.

- Fluencia verbal fonémica. Valora la capacidad para producir palabras de acuerdo con una cate- 
Tabla I. Datos demográficos del grupo clínico y del grupo control.

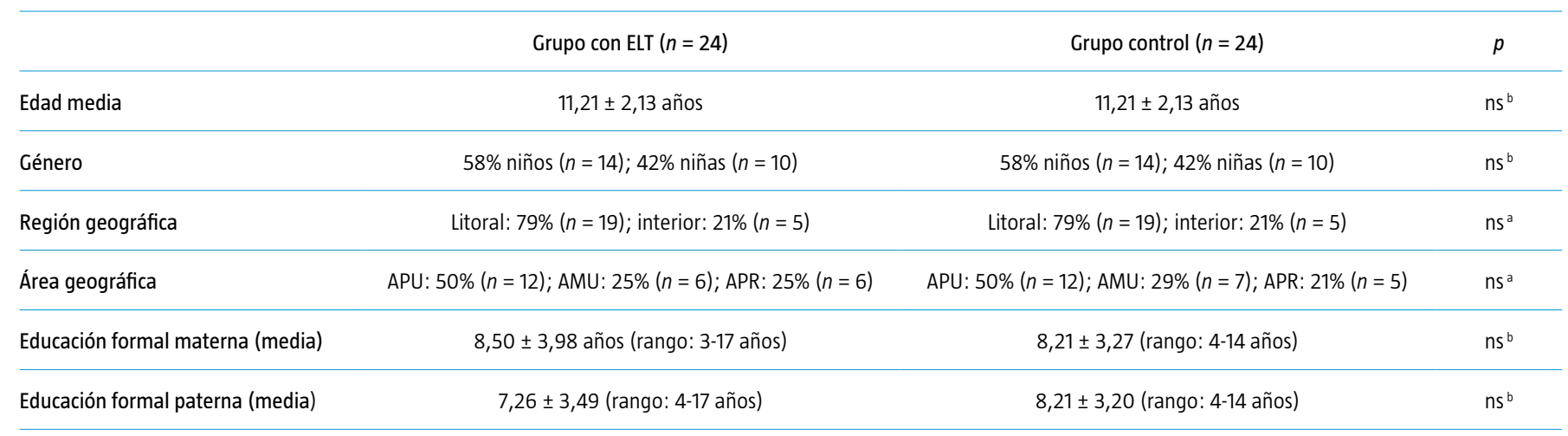

AMU: área moderadamente urbana; APR: área predominantemente rural; APU: área predominantemente urbana; ns: no significativo. ${ }^{\mathrm{a}} \chi^{2}$; ${ }^{\mathrm{b}}$ Test de Mann-Whitney.

goría fonémica y el sujeto debe decir un máximo de palabras que comienzan por una letra determinada ( $P, M$ y $R$ ) en un minuto para cada letra.

El grupo clínico consiste en 24 sujetos afectos de epilepsia del lóbulo temporal (14 niños y 10 niñas). Todos los niños con epilepsia que entraron en el estudio reunían los siguientes criterios:

- Resultados $\geq 70$ en al menos uno de los cocientes intelectuales (CI) del WISC-III.

- Estar entre 7 y 15 años de edad.

- Sin diagnóstico previo de encefalitis ni meningitis.

- Tomar uno o dos fármacos o haber suspendido la medicación.

- Diagnosticado de epilepsia del lóbulo temporal.

El grupo de control comporta el mismo número de pacientes que se adecuaron al grupo clínico en edad, género, zona de residencia y nivel escolar de sus padres. La tabla I resume las características demográficas de ambos grupos.

Se recogió de cada niño la información clínica siguiente: etiología, tipos de crisis, edad de comienzo, evolución de la epilepsia y medicación (Tablas II y III). La clasificación de las crisis y del tipo de epilepsia se hizo de acuerdo con el sistema de clasificación de la Liga Internacional contra la Epilepsia [31,32]. Así, el diagnóstico de crisis epiléptica se basa en la historia clínica (todos los niños tienen clínica compatible con la semiología de las crisis del lóbulo temporal) y en el videoelectroencefalograma intercrítico (con actividad paroxística localizada en el lóbulo temporal). Todos los sujetos tienen estudio de imagen.

En la tabla II presentamos los resultados obtenidos con el WISC-III. En primer lugar, queremos subrayar un valor por debajo del promedio del CI en la escala total (media: $83,75 \pm 15,33$ ), considerando el valor de la media en alguna parte entre 90 y 109 . Estos resultados son consistentes con los encontrados en otros estudios [2,33-35]. Queremos destacar las dificultades registradas en la velocidad de procesamiento, teniendo en cuenta que el rendimiento en el índice de velocidad de procesamiento es el resultado compuesto del WISC-III con puntuaciones más bajas.

Respecto a la situación académica del grupo de niños con epilepsia, pudimos comprobar que 8 niños (33\%) habían tenido que repetir un año en la escuela y que 11 (46\%) precisaban educación especial.

\section{Resultados}

En la tabla IV comparamos resultados obtenidos por los dos grupos (epilepsia de lóbulo temporal y grupo control) en tareas de atención y de funciones ejecutivas. En la prueba de cancelación (evaluación de la atención sostenida) encontramos diferencias entre grupos en la forma de tres signos (10 a 15 años) tanto en el resultado global $(U=86,5 ; p=0,002)$ como en el número de símbolos que fueron cancelados correctamente $(U=100 ; p=0,007)$. En la prueba asignada a niños más pequeños de dos signos, encontramos la misma tendencia (resultado global: $U=2 ; p=0,081$; signos correctos: $U=2 ; p=0,083$ ), aunque sin significancia estadística. Los resultados para la evaluación de la atención selectiva (Trail Making Test, parte A) están significativamente por debajo en el grupo con epilepsia del lóbulo temporal $(U=115,5 ; p=0,002)$ y en la parte B de esta prueba, que intenta evaluar la atención dividida $(U=127$; $p=0,002)$. En los resultados relativos a omisiones y errores, no hay diferencias entre los grupos en nin- 
Tabla II. Datos clínicos y funcionamiento intelectual del grupo con epilepsia del lóbulo temporal.

\begin{tabular}{|c|c|}
\hline \multicolumn{2}{|l|}{ Etiología } \\
\hline Idiopática & $13 \%(n=3)$ \\
\hline Sintomática & $29 \%(n=7)$ \\
\hline Criptogénica & $58 \%(n=14)$ \\
\hline \multicolumn{2}{|l|}{ Tipo de crisis } \\
\hline Parcial simple & $8 \%(n=2)$ \\
\hline Parcial compleja & $42 \%(n=10)$ \\
\hline Parcial secundariamente generalizada & $50 \%(n=12)$ \\
\hline \multicolumn{2}{|l|}{ Edad de comienzo } \\
\hline$\leq 5$ años & $42 \%(n=10)$ \\
\hline$\geq 6$ años & $58 \%(n=14)$ \\
\hline \multicolumn{2}{|l|}{ Evolución } \\
\hline Sin crisis & $54 \%(n=13)$ \\
\hline Con crisis & $46 \%(n=11)$ \\
\hline \multicolumn{2}{|l|}{ Medicación } \\
\hline Sin medicación & $4 \%(n=1)$ \\
\hline Monoterapia & $88 \%(n=21)$ \\
\hline Politerapia & $8 \%(n=2)$ \\
\hline \multicolumn{2}{|l|}{ WISC-III (media \pm desviación estándar) } \\
\hline Cociente intelectual verbal & $86,58 \pm 14,74$ \\
\hline Cociente intelectual manipulativo & $86,87 \pm 13,78$ \\
\hline Cociente intelectual total & $83,75 \pm 15,33$ \\
\hline Índice de comprensión verbal & $87,63 \pm 14,15$ \\
\hline Índice de organización perceptiva & $92,00 \pm 14,11$ \\
\hline Índice de rapidez de procesamiento & $81,00 \pm 14,01$ \\
\hline
\end{tabular}

WISC-III: test de inteligencia para niños de Wechsler, tercera edición.

guno de estos test (test de cancelación y Trail Making Test). Esto nos lleva a preguntarnos si los resultados más bajos obtenidos no fueron consecuencia de un rendimiento inferior más que de un déficit de atención real. En la prueba que intenta valorar las funciones ejecutivas (Torre de Londres), el análisis estadístico solamente encuentra diferencias significativas en errores de tipo II $(U=214 ; p=0,032)$. En la prueba de fluencia verbal fonémica, el análisis estadístico muestra una diferencia significativa entre ambos grupos $(U=184 ; p=0,031)$.
Tabla III. Medicación y dosis del grupo con epilepsia del lóbulo temporal.

\begin{tabular}{|c|c|c|}
\hline Pacientes & Edad (años) & Medicación y dosis \\
\hline 1 & 7 & Ácido valproico (800 mg/día) \\
\hline 2 & 7 & Lamotrigina (200 mg/día) \\
\hline 3 & 8 & Ácido valproico (600 mg/día) \\
\hline 4 & 9 & $\begin{array}{l}\text { Ácido valproico }(1.000 \mathrm{mg} / \text { día}) \\
\text { Levetiracetam }(1.500 \mathrm{mg} / \mathrm{dí})\end{array}$ \\
\hline 5 & 10 & Ácido valproico (500 mg/día) \\
\hline 6 & 10 & Ácido valproico (1.000 mg/día) \\
\hline 7 & 10 & Ácido valproico (300 mg/día) \\
\hline 8 & 11 & Ácido valproico (1.000 mg/día) \\
\hline 9 & 11 & Sin medicación \\
\hline 10 & 11 & Ácido valproico (1.000 mg/día) \\
\hline 11 & 11 & Ácido valproico (500 mg/día) \\
\hline 12 & 11 & Ácido valproico (1.000 mg/día) \\
\hline 13 & 11 & $\begin{array}{l}\text { Ácido valproico }(1.600 \mathrm{mg} / \mathrm{día}) \\
\text { Carbamacepina }(200 \mathrm{mg} / \mathrm{dí})\end{array}$ \\
\hline 14 & 11 & Ácido valproico (600 mg/día) \\
\hline 15 & 12 & Carbamacepina (400 mg/día) \\
\hline 16 & 12 & Ácido valproico (1.000 mg/día) \\
\hline 17 & 12 & Ácido valproico (800 mg/día) \\
\hline 18 & 12 & Ácido valproico (1.000 mg/día) \\
\hline 19 & 13 & Topiramato (175 mg/día) \\
\hline 20 & 13 & Carbamacepina (600 mg/día) \\
\hline 21 & 13 & Ácido valproico (1.100 mg/día) \\
\hline 22 & 14 & Ácido valproico (1.500 mg/día) \\
\hline 23 & 15 & Ácido valproico (1.000 mg/día) \\
\hline 24 & 15 & Ácido valproico (1.000 mg/día) \\
\hline
\end{tabular}

Comprobamos en la bibliografía revisada la tendencia a un mayor riesgo de presentar deficiencias cognitivas en aquellos pacientes cuya edad de comienzo de la epilepsia es precoz [36-39]. Esto nos 
Tabla IV. Resultados en atención y tareas de funciones ejecutivas. Comparación entre grupos de epilepsia del lóbulo temporal (ELT) y grupo control.

\begin{tabular}{|c|c|c|c|c|c|c|c|c|}
\hline & \multicolumn{3}{|c|}{ Grupo ELT $(n=24)$} & \multicolumn{3}{|c|}{ Control $(n=24)$} & \multirow[b]{2}{*}{$U$} & \multirow[b]{2}{*}{$p$} \\
\hline & Media & DE & Rango & Media & $\mathrm{DE}$ & Rango & & \\
\hline \multicolumn{9}{|l|}{ Test de cancelación } \\
\hline Dos señales (7-9 años) ${ }^{a}$ & 6,25 & 4,57 & $1-12$ & 10,75 & 3,2 & $8-14$ & 2 & 0,081 \\
\hline Dos señales: signos correctos ${ }^{\mathrm{a}, \mathrm{b}}$ & 63 & 30,41 & $25-95$ & 107,25 & 31,4 & $80-152$ & 2 & 0,083 \\
\hline Dos señales: omisiones ${ }^{\mathrm{a}, \mathrm{b}}$ & 11 & 6,83 & $2-18$ & 18 & 9,13 & $7-29$ & 4 & 0,248 \\
\hline Dos señales: errores ${ }^{a, b}$ & 3 & 4,69 & $0-10$ & 0,25 & 0,5 & $0-1$ & 3,5 & 0,155 \\
\hline Tres señales (10-15 años) c & 7,55 & 4,44 & $1-18$ & 11,55 & 2,8 & $7-17$ & 86,5 & 0,002 \\
\hline Tres señales: signos correctos ${ }^{\mathrm{b}, \mathrm{c}}$ & 160,6 & 57,22 & $66-300$ & 205,3 & 50,09 & $132-325$ & 100 & 0,007 \\
\hline Tres señales: omisiones ${ }^{b, c}$ & 17,95 & 21,35 & $1-88$ & 15,2 & 9,25 & $1-41$ & 175 & 0,498 \\
\hline Tres señales: errores ${ }^{b, c}$ & 0,7 & 0,92 & $0-3$ & 0,45 & 0,83 & $0-3$ & 168 & 0,316 \\
\hline \multicolumn{9}{|l|}{ Trail Making Test } \\
\hline Trail A: tiempo & 7,13 & 3,38 & $1-13$ & 10,79 & 3,26 & $1-17$ & 115,5 & 0,002 \\
\hline Trail A: errores ${ }^{b}$ & 0,08 & 0,28 & $0-1$ & 0,08 & 0,28 & $0-1$ & 288 & 0,137 \\
\hline Trail B: tiempo ${ }^{d}$ & 6,68 & 4,02 & $1-13$ & 10,45 & 2,61 & $4-14$ & 127 & 0,002 \\
\hline Trail B: errores ${ }^{b, d}$ & 0,86 & 1,52 & $0-5$ & 0,18 & 0,39 & $0-1$ & 210,5 & 0,137 \\
\hline \multicolumn{9}{|l|}{ Torre de Londres } \\
\hline Primer ensayo & 8,83 & 3,84 & $3-16$ & 9,75 & 2,74 & $5-15$ & 232,5 & 0,249 \\
\hline Total & 8,92 & 3,17 & $1-11$ & 10,17 & 2,08 & $3-12$ & 217,5 & 0,094 \\
\hline Número de ensayos & 8,29 & 4,01 & $1-16$ & 9,88 & 3,25 & $5-15$ & 219 & 0,153 \\
\hline Errores de tipo I & 0,17 & 0,48 & $0-2$ & - & - & - & 252 & 0,077 \\
\hline Errores de tipo II & 1,29 & 2,53 & $0-9$ & 0,17 & 0,56 & $0-2$ & 214 & 0,032 \\
\hline Errores de tipo III & 7,33 & 3,87 & $1-14$ & 6,29 & 2,9 & $2-11$ & 242 & 0,341 \\
\hline Fluencia verbal fonémica & 7,75 & 2,88 & $3-16$ & 9,71 & 3,2 & 4-15 & 184 & 0,031 \\
\hline
\end{tabular}

DE: desviación estándar. ${ }^{a}$ Grupo de ELT: $n=4$; control: $n=4$; ${ }^{b}$ Para estos resultados se consideran resultados brutos. ${ }^{\mathrm{c}}$ Grupo ELT: $n=20$; control: $n=20$; ${ }^{d}$ Grupo ELT: $n=22$; control: $n=22$ (porque dos niños no conocían el alfabeto).

ha llevado a valorar si existen diferencias de rendimiento en las pruebas neuropsicológicas analizadas entre dos grupos: a) grupo con epilepsia de lóbulo temporal con comienzo precoz ( $\leq 5$ años de edad), y b) grupo de epilepsia con comienzo más tardío $(\geq 6$ años de edad). Como regla general, observamos una tendencia a un rendimiento más pobre en niños y adolescentes cuya edad de comienzo de su epilepsia es inferior a 6 años, comparados con los resultados obtenidos con edades superiores. De todos modos, estas diferencias sólo tenían significación estadística en el test de la Torre de Londres (problemas correctos en el primer ensayo: $U=28 ; p=0,013$; número de ensayos: $U=34 ; p=0,036$ ) y en el test de cance- lación de tres señales (puntuación total: $U=7 ; p=$ 0,001 ; signos correctos: $U=12,5 ; p=0,006$ ).

Cuando comparamos los grupos con crisis y el grupo libre de ellas durante al menos seis meses, el primero presenta resultados promedios inferiores en la mayoría de pruebas. De todas formas, esta diferencia sólo fue estadísticamente significativa en la prueba de fluencia verbal fonémica $(U=21,5 ; p=0,002)$.

\section{Discusión}

En el área de la atención se observaron déficit en las tareas de atención sostenida, atención selectiva y 
atención dividida, pese a que el rendimiento de los sujetos con epilepsia del lóbulo temporal sólo diferían significativamente de los del grupo control en los valores relacionados con el tiempo necesario para completar las tareas y no en la realización de omisiones o errores. Esto puede ser consecuencia de un déficit de la velocidad de procesamiento, posiblemente anterior a los déficit en el área de la atención [40]. Este proceso de enlentecimiento puede afectar las capacidades de atención de estos niños y adolescentes. Por otra parte, las dificultades mostradas en el índice de velocidad de procesamiento del WISC-III (véase 'Sujetos y métodos') añaden peso a la hipótesis de que los niños y adolescentes con epilepsia del lóbulo temporal tienen problemas en la velocidad de procesamiento. Por otro lado, debemos considerar el efecto de los fármacos antiepilépticos en la velocidad de procesamiento $[41,42]$. En un estudio de Hessen et al [43], los niños con epilepsia fueron evaluados en tareas neuropsicológicas antes y después de la interrupción de la medicación antiepiléptica, y se observó que después de la interrupción el desempeño de los sujetos mejoró significativamente en las tareas más exigentes desde el punto de vista de la velocidad de procesamiento.

Por tanto, la explicación de las dificultades mostradas en la prueba de fluencia verbal fonémica puede no deberse a un trastorno de las funciones ejecutivas, sino a alteraciones de la velocidad de procesamiento, así como del lenguaje [44]. Debemos resaltar el hecho de que un test no valora solamente funciones primarias, sino también áreas secundarias. Por ejemplo, los problemas de memoria valorados mediante problemas de evaluación de memoria pueden ser secundarios a déficit de otros campos, como la atención, las funciones ejecutivas, el lenguaje, las capacidades visuoperceptivas e incluso factores emocionales. De ahí la necesidad de ampliar los protocolos de evaluación neuropsicológica a cada dimensión de la función neurocognitiva; debemos tener en cuenta que ningún test valora una sola función: primero, la misma clasificación de los instrumentos para valorar cada área todavía genera controversia, como, por ejemplo, la prueba de fluencia verbal fonémica, que puede clasificarse como un test de lenguaje [12] o como un test de funciones ejecutivas [16]; y segundo, más allá de esta categorización, la tarea en sí pude exigir, como ya se ha mencionado, la movilización de diferentes funciones. Este aspecto debe tenerse en consideración cuando se interpreten los resultados.

Desde el punto de vista del comienzo de la epilepsia, los niños que la inician precozmente muestran mayor dificultad en el mantenimiento de la atención y en la capacidad de planificación. Por ello, los déficit de atención y de control ejecutivo en este grupo de niños con epilepsia del lóbulo temporal parecen estar asociados con epilepsias cuyo inicio tiene lugar antes de los 6 años de edad.

La existencia de déficit de la atención y de las funciones ejecutivas genera dificultades de funcionamiento en estos niños -que van desde las actividades rutinarias básicas a los procesos complejos del aprendizaje-, debido al impacto que tienen sobre las capacidades metacognitivas, básicas para el control de la actividad cognitiva [45]. A consecuencia de ello, tiene un efecto desfavorable sobre la calidad de vida, y afecta principalmente al bienestar psicológico, social y educativo. Estos resultados indican la necesidad de valorar y monitorizar las áreas de atención, de las funciones ejecutivas y de la velocidad de procesamiento de la información en los niños y adolescentes con epilepsia del lóbulo temporal, especialmente en aquéllos en que la edad de comienzo de la epilepsia es precoz. Estas dificultades parecen contribuir significativamente al fracaso escolar. Por ello, una intervención enfocada a paliar estos problemas puede ser de gran trascendencia. El tratamiento puede incluir terapia cognitivoconductual (por ejemplo, entrenamiento de los padres, intervención en la escuela para facilitar estructura y retroalimentación frecuente e inmediata) y terapia farmacológica (psicoestimulantes).

\section{Bibliografía}

1. Alonso E, Álvarez M, Reyes A, Rojas E, Romero C, Pando A. Trastornos de atención en una muestra de niños con epilepsia parcial compleja. Rev Neurol 2001; 33: 991-3.

2. Auclair L, Jambaqué I, Olivier D, David L, Eric S. Deficit of preparatory attention in children with frontal lobe epilepsy. Neuropsychologia 2005; 43: 1701-12.

3. Culhane-Shelburne K, Chapieski L, Hiscock M, Glaze D. Executive functions in children with frontal and temporal lobe epilepsy. J Int Neuropsychol Soc 2002; 8: 623-32.

4. Henkin Y, Sadeh M, Kivity S, Shabtai E, Rabin L, Gadoth $\mathrm{N}$. Cognitive function in idiopathic generalized epilepsy of childhood. Dev Med Child Neurol 2005; 47: 126-32.

5. Williams J. Learning and behavior in children with epilepsy. Epilepsy Behav 2003; 4: 107-11.

6. Lopes A. Crianças e adolescentes com epilepsia: estudos com a bateria de avaliação neuropsicológica de Coimbra [tese]. Coimbra: Faculdade de Psicologia e de Ciências da Educação da Universidade de Coimbra; 2007.

7. Dunn D, Kronenberger W. Childhood epilepsy, attention problems, and ADHD: review and practical considerations. Pediatr Neurol 2006; 12: 222-8.

8. Anderson V, Northam E, Hendy J, Wrennall J. Developmental neuropsychology: a clinical approach. Philadelphia: Psychology Press; 2001.

9. Deonna T, Roulet-Perez E. Cognitive and behavioural disorder of epileptic origin in children. London: Mac Keith Press; 2005.

10. Hessen E, Lossius M, Reinvang I, Gjerstad L. Influence of major antiepileptic drugs on attention, reaction time, and speed information processing: results from a randomised, 
double-blind, placebo-controlled withdrawal study of seizure-free epilepsy patients receiving monotherapy. Epilepsia 2006; 47: 2038-45.

11. Alberto I. Atenção, por favor!: à avaliação da atenção. Psychologica 2003; 34: 231-43.

12. Lezak M, Howieson D, Loring D. Neuropsychological assessment, 4 ed. New York: Oxford University Press; 2004.

13. Linden M, Meulemans T, Seron X, Coyette F, Andrés P, Prairial C. L'évaluation des fonctions exécutives. En Seron $\mathrm{X}$, Linden M, eds. Traité de neuropsychologie clinique. Marseille: Solal; 2000. p. 275-300.

14. Pereira M, Gaspar M, Simões M, Lopes A. Funções executivas: uma nova metodologia de avaliação do comportamento inteligente. Sobredotação 2006; 7: 177-86.

15. Papazian O, Alfonso I, Luzondo R, Araguez N. Entrenamiento de la función ejecutiva en preescolares con trastorno por déficit de atención/hiperactividad combinado: estudio prospectivo, controlado y aleatorizado. Rev Neurol 2009; 48 (Supl 2): S119-22.

16. Baron I. Neuropsychological evaluation of the child. New York: Oxford University Press; 2004.

17. Williams J, Phillips T, Griebel M, Sharp G, Lange B, Edgar $\mathrm{T}$, et al. Factors associated with academic achievement in children with controlled epilepsy. Epilepsy Behav 2001; 2: 217-23.

18. Aldenkamp A, Reijs R, Mil V, Hall M. Attention disorders in children with epilepsy. Epilepsia 2005; 46 (Suppl 6): S6.

19. Martín-González R, González-Pérez P, IzquierdoHernández M, Hernández-Expósito $\mathrm{S}$, Alonso-Rodríguez M, Quintero-Fuentes I, et al. Evaluación neuropsicológica de la memoria en el trastorno por déficit de atención/ hiperactividad: papel de las funciones ejecutivas. Rev Neurol 2008; 47: 225-30.

20. Fabre L, Valdes R, García R. Attention evaluation in children with idiopathic temporal lobe epilepsy. Epilepsia 2005; 46 (Suppl 6): S222.

21. Fleck D, Shear P, Straskowski S. A reevaluation of sustained attention performance in temporal lobe epilepsy. Arch Clin Neuropsychol 2002; 17: 399-405.

22. Guimarães C, Li L, Rzezak P, Fuentes D, Franzon R, Montenegro M, et al. Temporal lobe epilepsy in childhood: comprehensive neuropsychological assessment. J Child Neurol 2007; 22: 836-40.

23. Igarashi K, Oguni H, Osawa M, Awaya Y, Kato M, Mimura $\mathrm{M}$, et al. Wisconsin Card Sorting Test in children with temporal lobe epilepsy. Brain Dev 2002; 24: 174-8.

24. Laurent A, Arzimanoglou A. Cognitive impairments in children with nonidiopathic temporal lobe epilepsy. Epilepsia 2006; 47 (Suppl 2): S99-102.

25. Rzezak P, Fuentes D, Guimarães C, Thome-Souza S, Kuczynski E, Li L, et al. Frontal lobe dysfunction in children with temporal lobe epilepsy. Pediatr Neurol 2007; 37: 176-85.

26. García-Peñas J. Autismo, epilepsia y patología del lóbulo temporal. Rev Neurol 2009; 48 (Supl 2): S35-45.

27. Corcoran R, Upton D. A role for the hippocampus in card sorting? Cortex 1993, 29: 293-304.

28. García-Molina A, Enseñat-Cantallops A, Tirapu-Ustárroz J, Roig-Rovira T. Maduración de corteza prefrontal y desarrollo de las funciones ejecutivas durante los primeros cinco años de vida. Rev Neurol 2009, 48: 435-40.

29. Wechsler D. Escala de inteligência de Wechsler para crianças, terceira edição (WISC-III): manual. Lisboa: Cegoc; 2003.
30. Simões M, Albuquerque C, Pinho S, Pereira, M, SeabraSantos M, Alberto I, et al. Relatório do projeto adaptação e aferição de testes neuropsicológicos: estudos psicométricos. Coimbra: Faculdade de Psicologia e de Ciências da Educação da Universidade de Coimbra; 2008.

31. Commission on Classification and Terminology of the International League Against Epilepsy. Proposal for revised clinical and electroencephalographic classification of epileptic seizures. Epilepsia 1981; 22: 489-501.

32. Commission on Classification and Terminology of the International League Against Epilepsy. Proposal for revised classification of epilepsies and epileptic syndromes. Epilepsia 1989; 30: 389-99.

33. Aldenkamp A, Weber B, Wihelmina C, Overweg-Plandsoen W, Reijs R, Mil S. Educational underachievement in children with epilepsy: a model to predict the effects of epilepsy on educational achievement. J Child Neurol 2005; 20: 175-80.

34. Hernandez M, Sauerwein H, Jambaqué I, Guise E, Lussier F, Lortie A, et al. Deficits in executive functions and motor coordination in children with frontal lobe epilepsy. Neuropsychologia 2002; 40: 384-400.

35. Williams J, Griebel L, Dykman R. Neuropsychological patterns in pediatric epilepsy. Seizure 1998; 7: 223-8.

36. Beckung E, Uvebrant P. Hidden dysfunction in childhood epilepsy. Dev Med Child Neurol 1997; 39: 72-9.

37. Cormack F, Cross J, Isaacs E, Harkness W, Wright I, VarghaKhadem F, et al. The developmental of intellectual abilities in pediatric temporal lobe epilepsy. Epilepsia 2007; 48: 201-4.

38. Riva D, Saletti V, Nichelli F, Bulgheroni S. Neuropsychologic effects of frontal lobe epilepsy in children. J Child Neurol 2002; 17: 661-7.

39. Sillanpää M. Learning disability: occurrence and long-term consequences in childhood-onset epilepsy. Epilepsy Behav 2004; 5: 937-44.

40. Hernández M, Sauerwein H, Jambaqué I, Guise E, Lussier F, Lortie A, et al. Attention, memory and behavioural adjustment in children with frontal lobe epilepsy. Epilepsy Behav 2003; 4: 522-36.

41. Aldenkamp A. Cognitive side-effects of antiepileptic drugs. In Jambaqué I, Lassonde M, Dulac O, eds. Neuropsychology of childhood epilepsy. New York: Kluver Academic/Plenum Publishers; 2001. p. 257-67.

42. Vermeulen J, Kortstee, S, Alpherts W, Aldenkamp A. Cognitive performance in learning disabled children with and without epilepsy. Seizure 1994; 3: 13-21.

43. Hessen E, Lossius M, Reinvang I, Gjerstad L. Influence of major antiepileptic drugs on attention, reaction time, and speed information processing: results from a randomised, double-blind, placebo-controlled withdrawal study of seizure-free epilepsy patients receiving monotherapy. Epilepsia 2006; 47: 2038-45.

44. Sauerwein H, Gallagher A, Lassonde M. Neuropsychological deficits in children with temporal lobe epilepsy. In Arzimanoglou A, Aldenkamp A, Cross H, Lassonde M, Moshé S, Schmitz $B$, eds. Cognitive dysfunction in children with temporal lobe epilepsy. Esher: John Libbey Eurotext; 2005. p. 1-12.

45. Riva D, Avanzini G, Franceschetti S, Nichelli F, Valetti V, Vago C, et al. Unilateral frontal lobe epilepsy affects executive functions in children. Neurol Sci 2005; 26: 263-70. 
Neuropsychological evaluation in children with epilepsy: attention and executive functions in temporal lobe epilepsy

Introduction. Attention and executive functions correspond to important areas of cognitive functioning associated with the frontal cortex. The study of attention and executive functions in children with epilepsy has focused on characterizing the group with frontal lobe epilepsy. Still, recent studies have identified deficits in these areas also in children with temporal lobe epilepsy (TLE).

Aim. To investigate attention and executive functions in a group of children with TLE, also considering the influence of clinical variables (age at onset of epilepsy and evolution of seizures).

Subjects and methods. Attention and executive functions were studied in a group of 24 children with TLE, aged 7-15 years and compared with 24 control children of the same age and socio-cultural level. Subjects were assigned the following tasks: Cancellation Task, Trail Making Test, Tower of London and Phonemic Verbal Fluency.

Results. The group with TLE performed significantly lower on selective, sustained and divided attention and on phonemic verbal fluency. In markers concerning commissions and omissions there were no differences between groups in any of the performed tests. A significant slowing of processing speed was reported. In addition, patients with earlier age at onset of epilepsy had more difficulties in sustained attention and planning abilities.

Conclusion. These results sustain the need for evaluating and monitoring the area of attention, executive functions and processing speed in children and adolescents with temporal lobe epilepsy, above all those with earlier age at onset of epilepsy.

Key words. Attention. Cancellation Task. Children. Executive functions. Neuropsychological assessment. Phonemic Verbal Fluency. Temporal lobe epilepsy. Tower of London. Trail Making Test. 